\title{
Control Structure for Interpreting Handwritten Addresses
}

Edward Cohen, Jonathan J. Hull, and Sargur N. Srihari

\begin{abstract}
This correspondence describes the control structure for an intelligent handwritten address interpretation system. The system takes a grey-level address image, segments the address into lines and words, parses the address into meaningful syntactic categories, recognizes words using dynamically generated lexicons, and determines the destination code with the aid of postal directories.
\end{abstract}

Index Terms-Text processing, handwriting recognition, image processing

\section{INTRODUCTION}

The Handwritten Aojdress Integpretation System (HWAIS) rakes an off-line handwritten postal address image and determines a unique mail delivery point (e.g.. a mailbox). In the United States, each

Manuscrip received May 20. 1992: revised February 21, 1994. Recommended for aceptance by Associate Editor T. Deall.

F. Cohen is with Acer Sor Systems inc.. 5II School House Rond. Telford. PA 18969) USA: c-mail: cohenecis.udel.cdu.

J.J. Hull, and S N. Srihari are with the Center for Document Analysis and Recognition (CEDAR). State Universily of New York at Buffalo, 226 Bell Hall. Buffalo. NY 14260 USA: c-mail: hull(s)bufalocedu and srihari@cs.buffialo.edu.

IEEE Log Number $04(15251$. 


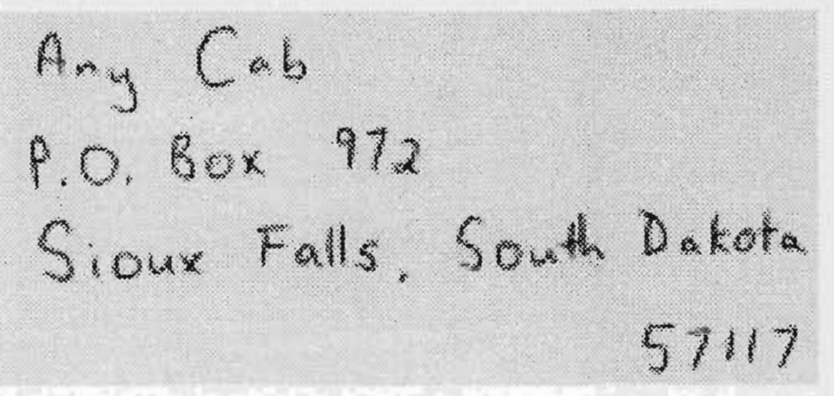

(a)

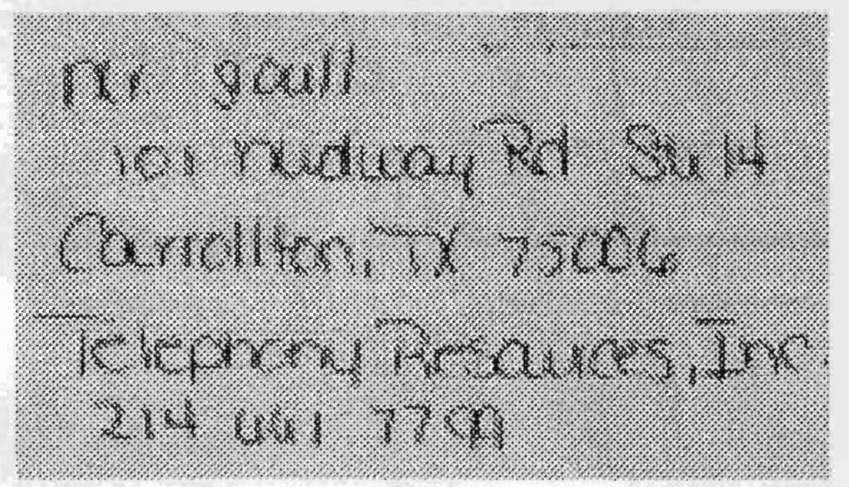

(c)

Fig. 1. Examples of handwrinten address blocks extracted from live mail

delivery point has heen assigned a number. a delivery point code (DPC), which is a ZIP Code with appended diegls. The IIWAIS must nonnally locate and read the ZIP Code and strect address or P.(). Box number to delermine the DPC. City and state names may be used to renolve ambiguties. Examples ol address laken from live mail (shown in lige. 1) illusmate some of the challenges. This correspondence oulines the IIW DSS contol smature with eximples. Sec [1]. [2] for gevater details and extensions to other domains.

The Unicd States Povial Service ilspSi funded the research described here (CFI) AR) and Imo smilar elforts (ERIM and CGA). While the onter groups had comparable performance of 7.IP code

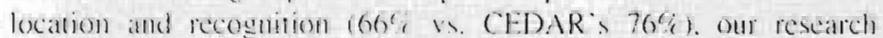
was the firs atempt to determine DPC from handwruten addresses whe other groups late non published figures for comparable sys (cms).

This correspondence is arganied as follows. Sections 11 describes algosthms. Section fll details how the system processes two handwriten address examples. Section IV describes performance evaluation and experimential resulis.

\section{HWISS ULGORTHM DESCRIPTION}

The control flow in the current HWAIS has ten operational stages.

1) Preprocessing:

(B) Texa line separation based on positional information separating an adtress inlo text lincs:

(i) Fature hembination:

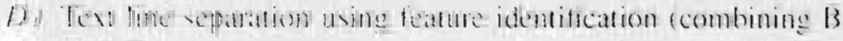
and $(\%$

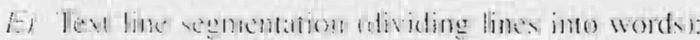

1.) Wond dansilication:

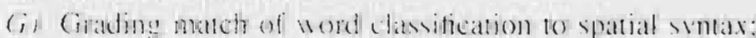

11. Recounding woth that hate a high symax-classilicaton makh:

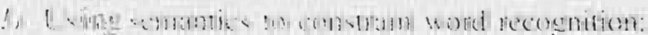

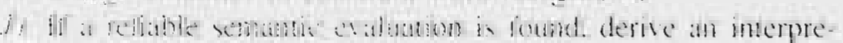

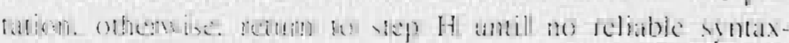

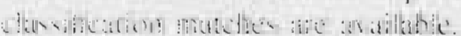

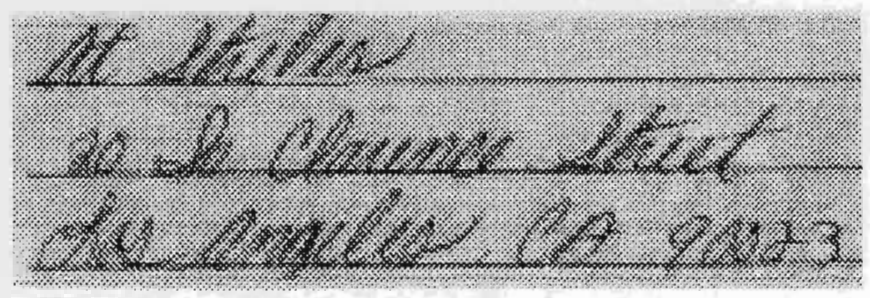

(b)

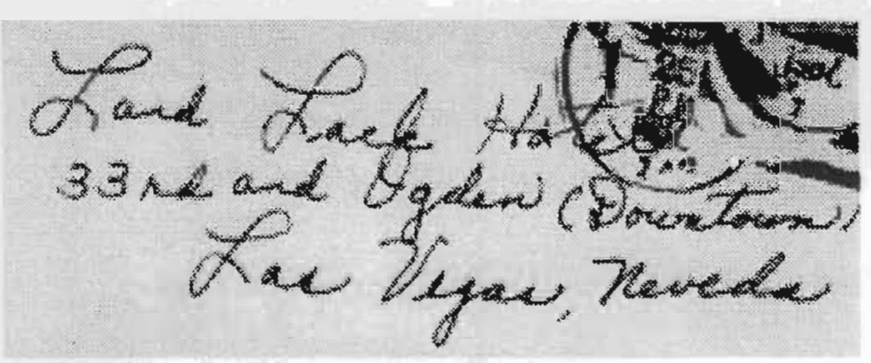

(d)

\section{Preprocessing}

(nput images are address blocks from live mail scanned at 300 pixels per inch with 256 shades of grey. Two preprocessing steps (thresholding |3| and guide line removal) are performed to produce a binary image (where texi pixels are black).

\section{Ininal tew line Soparaion}

Dividing the address into rex lines requires Iwo stages. Positional information separates "easy-10-place" components; stage D uses foature idemtitication to retine those results.

l.ne separation (Fig. 2) divides a binary image into verlical strips. Fach strip is converted to a sel of blocks. where blocks indicate the presence of ext. Overlapping blochs from adjacent strips are connected to fom a topological graph representing text lines (Fig. 3). where hlochs and bloch comncelions are represented as nodes and node connections respecrively. Heuristics adjust the graph to correct for typical siluations (c.g. ascenders).

After finalizing the block arrangement, connected components are assigned text lines based on their corresponding blocks. For example. a component located in blocks from a single lext is assigned to that line. A component s whose blocks overlap several text lines is assigned to a ser of lines. Small components (considered notse) are not assigned to lines. All line assigmments (including noise components) may be refined in sage $D$.

\section{Fenme demitu anim}

Feature identilicanon requites shape identilication and spatial confirmation. To improse speel. mow processing requiring image infor-

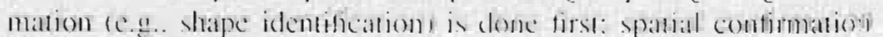
requires only symbotic intormation. Fach combected component is inibially identified as hatsing (or nor har ing) a particular shape (i.e.

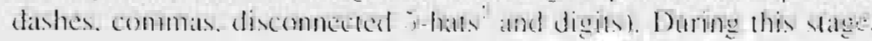

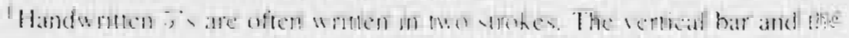

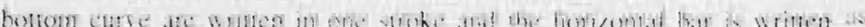

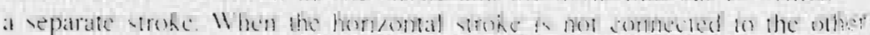

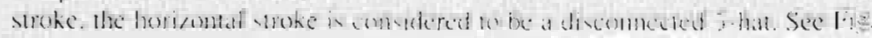
i! ii).
} 
Robert Sames

4woodsman Drive

Newarly Delaware 1971

Banjasmin

15

$7-$

Banjasrin

BLAIR NE 68009

Gripen

Indianapotis, Indiana

$N M_{82004}$ 
1052

IEEE TRANSACTIONS ON PATTERN ANALYSIS AND MACHINE INTELLIGENCE. VOL. 16, NO. 10, OCTOBER 1994

Clinton sora $52>36$

Chiton eros $52>36$

clinton sora 521736

Clinton sora 5:2>36

Clinton eroval5 $2>36$

Chin tor sora 521736

chiton eros $512>36$

clinton sora 512176

chiton Sowa15 2736

chiton soma 512136

Fig. 6. Different ways of separating a text lire into words. Two largest gaps give sixth grouping from lop. Correct choice (first and fourth larges gaps) is second from bottom.

Teat Line Se mentation

Components of each tex line are clustered into words 10 create different hypotheses. Words are formed by ordering a text line components from left to right and selecting word breaks between components. Word-brak locations at re hypothesized based on horizontal spaces between components. a shift in the mean vertical position of components. and the locution of commas (sec Fig. 5).

Word-break locations are ordered based on their distances (a comma-gap distance is computed as the sum of distances on enter side of the commas a shift in mean vertical position is always at word break). Different word-break groupings (Fig. () are chosen lo separate a line into 1-5 words (eeg. for three words. use the lop two widest word breaks for one set use first and third widest word breaks for another set).

Word Classification

Each word hypothesized by text line segmentation is classified (into one or more categories will assigned confidence values) based on features and spatial locations of its components. Word categoric are cancelers (including a mumber-of-chatacers estimate). (an state-abtheriation. digits (including a number-of-digits estimate)

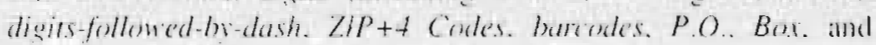
noise.

Grading Hond Categories a, Simar

Our stat is avo-dimensional. since words are horizontally orcered along a lex line and text lines are vertically ordered. One common handwritten address syntax has the botom-mose bine con tanning three words. city, sate. and Z.IP Code (CSZ). For this w max to be present the bottommost line must be div isible into be word and each must be matched to its expected form. The his word mus
TABLE I

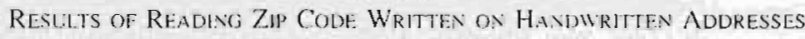

\begin{tabular}{|c|r|r|}
\hline Correctly read images found in the USPS directory & 746 & $74.0 \%$ \\
\hline Correctly read images not found in the USPS directory & 18 & $1.8 \%$ \\
\hline Total Correct & 764 & $\mathbf{7 5 . 8 0 \%}$ \\
\hline \hline Rejected images that contained ZIP Codes & 221 & $21.9 \%$ \\
\hline Rejected images that contained no ZIP Code & 11 & $2.2 \%$ \\
\hline Total Reject & 232 & $23.0 \%$ \\
\hline \hline Errors in images that contained ZIP Codes & 11 & $1.1 \%$ \\
\hline Errors in images that did not contain ZIP Codes & 1 & $0.1 \%$ \\
\hline Total Error & 12 & $1.2 \%$ \\
\hline \hline Total images & $\mathbf{1 0 0 8}$ & $100.0 \%$ \\
\hline
\end{tabular}

have characteristics of a "city" word. meaning it must be classified as a "city" or "text", but the "city" classification would have a higher confidence match. State name and ZIP Code are matched similarly. Matches between image text and syntax are graded by ranking high-confidence syntax matches with a highly probable syntax over low-confidence syntax matches with less probable syntax. The HWAIS system marches against five different syntaxes: $P . O+B o$ + number (all on one line), strect-number + street-nume (all on one line). ZIP Code (on 1-2 lines. 5-digit ZIP Code and 4-digit add-on may be on separate lines), city name + state name + ZIP Code (on 1 i lines). and state name + ZIP Code ton 1-3 lines). The HWAlS tries to find the most complete match possible (egg.. a city name + state name + ZIP Code match will be chosen over a ZIP Code match). Recognizing Words

Numbers (7.IP Code, P.O. Box number or stree number) are segmented into digits and recognized. For stale -abbreviations, a scgment-and-classify algorithm designed for characters is used: and for other word types, a dictionary is used to assist in recognition. See 11 for details.

Using Semantic Information to Constrain Word Recognition

Once all relevant words have passed through the initial recognition phase. semantic information is used to improve recognition performince. ZIP Codes are checked against at USPS directory. Only $44 \%$ of possible 5-digi ZIP Codes are assigned; so roughly $56 \%$ of errors are detected.

If the state name is recognized, it is compared to the first wo ZIP Code digits and several actions can be taken. When state name and ZIP Code agree. the ZIP Could is accepted as correct even if digits are recognized with low confidence. If the state name is recognized with high confidence and the firs and/or second digit of the ZIP Code do not have high-conlidence recognition. the Z.IP Code will be corrected to correspond w the state name if possible. If the state name and ZIP Code are recognized with high confidence and they conflict, the ZIP Code is accepted as correct since its recognition is more reliable. The HWAIS uses information from stree numbers and P.O. Box numbers. If a 9-digit Z. lP Code is recognized. its 4-digit add-on can be confirmed by recognizing the P.O. Box number. If a 5 -digit ZIP Code is recognized its 4 -digit add -on can be determined by recognizing the P.O. Box number. The HWAIS can locate and recognize P.O. Box numbers in $57 \%$ of the images which contain them.

If a ZIP Cole and stree number of an address are determined. a dictionary of possible stree names is extracted from a USPS directory. When a street name is chosen. the HWAIS uses the recognized 7.IP Code and stree address (street number and name) (6) assign it 4 -digit add -on. Adding the last wo digits of the stree number to the ZIP +t Code creates the DPC. On addresses containing stree addresses whose ZIP Codes were automatically located ard

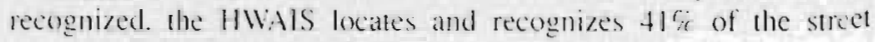
recognized. 


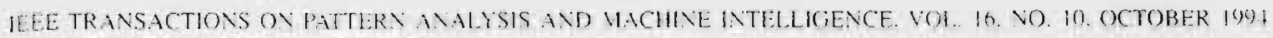

16153

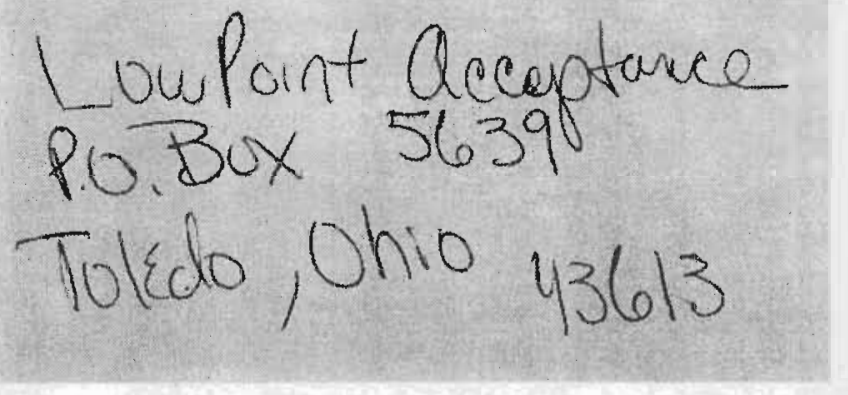

(a)

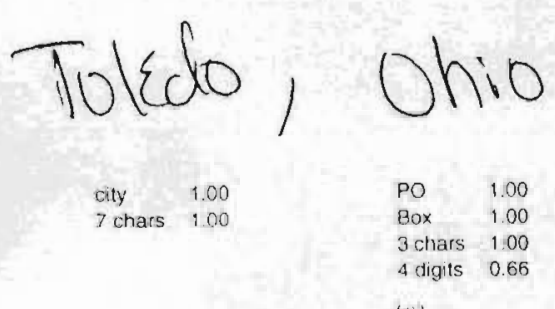

(i)

$\begin{array}{ll}\text { Original } & \text { Modified } \\ 40.299 & 40.999 \\ 30.988 & 30.988 \\ 60.997 & 60.997 \\ 10.994 & 10.994 \\ 30.994 & 30.994\end{array}$

(e)

$$
\begin{aligned}
& 50.996 \\
& 60.996 \\
& 30.999 \\
& 90.996
\end{aligned}
$$

(g)
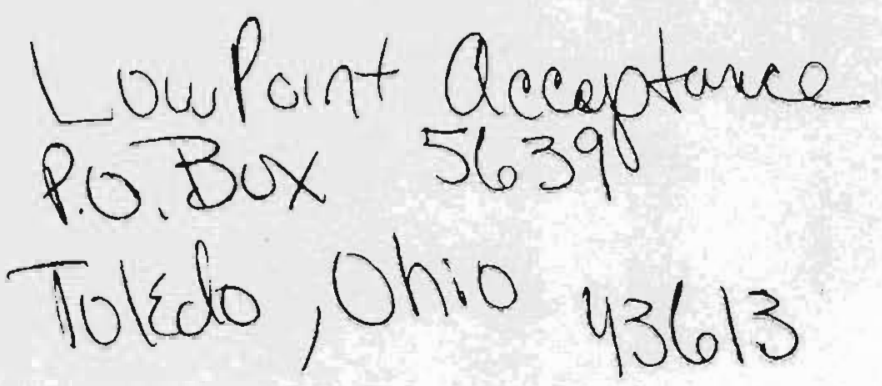

(b)

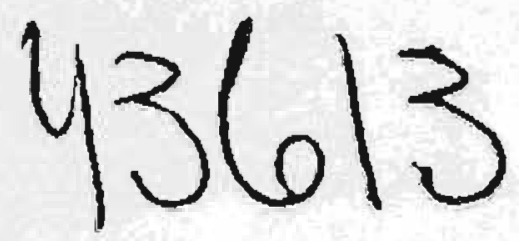

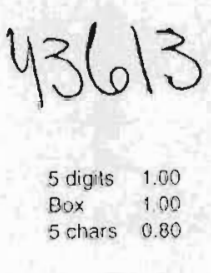

$\begin{array}{ll}2 \text { digits } & 1.00 \\ \text { Box } & 1.00 \\ \text { PO } & 0.80 \\ \text { state abrev } & 0.80 \\ 2 \text { chars } & 0.80\end{array}$
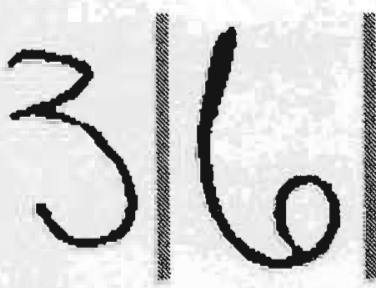

(d)
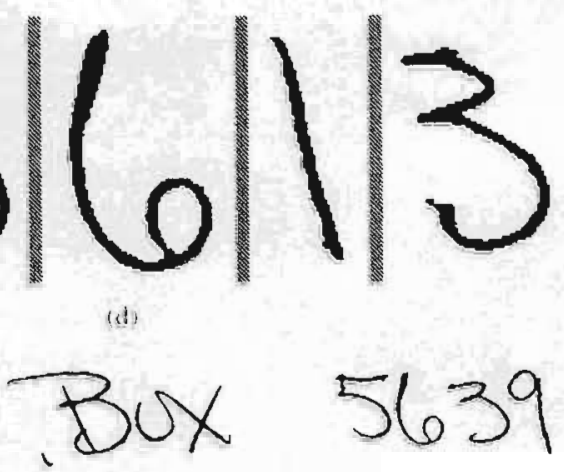

$\begin{array}{ll}\text { Box } & 1.00 \\ \text { state abbrev } & 1.00 \\ 2 \text { chars } & 1.00 \\ 2 \text { digits } & 0.34\end{array}$

(i)

Fig. 7. Example of HWAIS processing an image. (a) Grey-level image. (b) Thresholded image. (c) City, State. and ZIP (ode Candidates. (d) Segmented ZIP Code (e) Digits recognition results for Z.P Code (digit result and correct confidence) and adjusted digit recognition confidence results. (1) P.O.. Box. and Box-number Candidates. (g) Digit recognition results of P.O Box number. (h) 9-digit ZlP+4 Code (which is a DPC).

III. EXAMPLES OF HWAIS ON AN ADDRESS IMAGES

Figs. 7 and 8 show some intermediate steps of images processed by the HWAIS. The first example shows how a poorly recognized ZIP Code has its confidence increased by the state name recognition results and how the P.O. Box number is located and recognized to determine a DPC. The second example demonstrates how the IIWAIS can correctly locate a ZIP Code after initially failing to do so, and how a street address can determine a DPC.

The grey level for the first example (Fig. 7) is image shown in (a). and the thresholded image in (b). No guide line removal is required for this image; and, the address is separated into text lines and words.

Each text line is segmented imo several different word groupings. Word classifications for one three-word grouping of the line are shown in (c). The first word is classified as city (because it has text followed by a comma) and 7 -chars with high confidence (1.00). Similarly, the second word is classified as $P O$ (a short word with a component recognized as a zero at the end), $B$ or (a short word), and 3 (hats (the $i$ is considered to be noise) with high confidence. The word is also considered to be 4 -digits with moderate confidence $(0.66)$ because the first and last connected components are recognized as zeros.

This word segmentation results in a bottom-line syntax of city3-chars-5-digits (c) corresponding to cith-state-ZIP. Based on this match, the system selects the 5-digits word as the ZIP Code candidate. The 7.IP Code candidate is segmented (d) and isolated digits are recognized. Digit recognition results and their confidences are shown in (e). The recognized ZIP Code 43613 is checked against the USPS ZIP Code directory and found to be valid, but it would ordinarily be rejected because the first digit (which looks like a 4 or a 9 ) is recognized as a 4 with low confidence $(0.299)$. However, the state 
1054

IEEE TRANSACTIONS ON PATTERN ANALYSIS AND MACHINE INTELLIGENCE, VOL. 16, NO. 10, OCTOBER 1994

Dave Kibblehouse 318 E mam Str

Little Riven, The 01085 Little Riven, The 01085 $\operatorname{CASA}$
Pard Kibblehouse 318 E Main Str CASA (b)

1085

Little Riven, The 01085

$\begin{array}{ll}5 \text { chars } & 1.00 \\ 5 \text { digits } & 0.1\end{array}$

PO
state abbrev
3 chars
4 digits

10.993

50.438

20.910

50.990

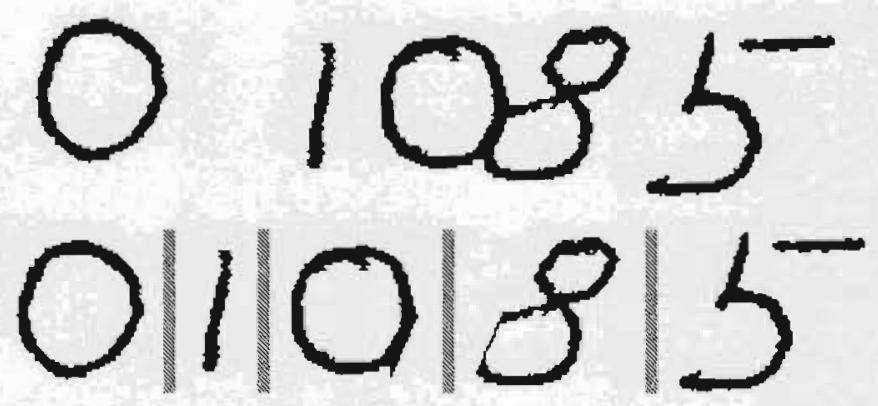

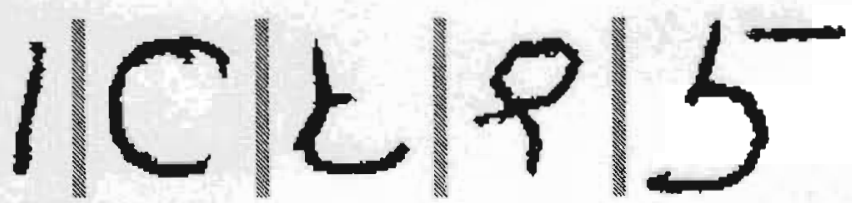

Little Riven, the 01085

00.992

10.993

00.993

80.995

Fig. 8. Example of HW: IS

$\sin$ (d) Fins segmented ZIP (Code. (c) Firs digits recognition results for ZIP Co

name is recognized as Ohio and since all Ohio ZIP Codes begin with

the digit 4 , the confidence of the firs digit is boosted. and the ZIIP code is accepted

The HWAS must also interpret the P.O. Box line 10 determine : DPC. The middle line of the address is parsed 10 P.O. -Bex-4-digits (i) which matches P.O.-Box-Bor-mmber. This line could also match 10 2-diens-2-ihars 4-chars for a street address line soreet-mumberweet-name ). but the P.O. Box line match hats higher confidence. The Box-number is recognized as 56.39 (g) wi high confidence and the t-digit add -on 0639 is determined using a lookup in a USPS the 9-digit DPC (h).

The second example (fig. 8) show the grey-level image (a) and thresholded image (b). Each tex line is separated and word polheses within each line are created $P$ for this editress the top parsing hypothesis for the ciry-state. bottom. One three-word hypothesis (c) for this line locates 5-charsstate-abbreviation-5-digits as a syntax match. This mach is incorrect. This mistake occurs because the ZIP Code contains a connected component (consisting of two large touching digits. 0 and 8 ) that is estimated to con ain three digits (making the lats word a 5 -digits
word). In addition. the middle word has (wo medium-sized connected 


\section{8 \\ 3 digits $\quad 1.00$ \\ Box $\quad 1.00$ \\ 3 chars 0.80
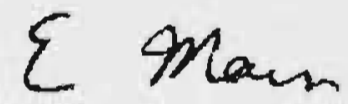 \\ 5 chars 0.67 \\ 8 digits 0.04 \\ (i)

$\begin{array}{lll}318 & \text { E MAIN ST } & -3311 \\ 318 & \text { E MOUNTAIN RD } & -1853 \\ 318 & \text { CITY VIEW BLVD } & -4006 \\ 318 & \text { LOCKHOUSE RD } & -1212\end{array}$

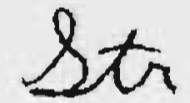

30.986

10.998

80.999

(1)
01085-3311

(m)

$\begin{array}{lll}318 & \text { CITY VIEW BLVD } & -4006 \\ 318 & \text { E MAIN ST } & -3311 \\ 318 & \text { E MOUNTAIN RD } & -1853 \\ 318 & \text { LOCKHOUSE RD } & -1212\end{array}$

(h)

\section{5-3311-18}

(ii)

Fig. 8. (conthued). Example of HWAIS processing an image. (i) Street number and name candidates. (j) Street number digit recognition results. (k) Original street name diclionary and 4-digil idelons. (1) Ranked street name dictionary and 4-digit addons. (m) 9-digit ZIP+4 Code. (n) Final DPC.

components which provides a good match to the state abbreviation classification.

The ZIP Code for this parse is segmented (d) and the digit results are shown in (e). Digit recognition results are not all high conlidence. and the state name is not recognized: so no digit corrections are made and the ZIP Code candidate is rejected

The second parsing hypothesis chosen for the cir-state-ZIP is the correct one. This parse of the same line into three words gives a syntax match of 5-chars-state-abbrevation-6-digirs. The 6-digits match to the ZIP Code is allowed because digit estimation wals required, and the HWAIS knows estimation emors may occur. The ZIP Cokle is segmented (g) and all digits are recognized with high confidence (h). The state name is still not recognized, so it does not affect the digit contidences.

The street address line (i) is correctly located by matching the second line, from the lop. 10 3-digits 5 -rhars. The street number is correctly recognized as 318 with high confidentce (j). Using a USPS directory, the street number, and the 5-digit ZIP Code, the HWAIS creates a dictionary ( $k$ ). This dictionary contains all valid street names in the 01805 7.P Code area whose street number is 318 . Their associated 4-digil add-ons (k) are also shown. The abbreviations in the street names are expanded (c.g.. E MAIN ST becomes EAST MAIN ST, EAST MAIN STR. EAST MAIN STREET, E MAIN ST. E MAIN STR, etc.) to create an expanded dictionary. This dictionary and the street name image are passed to the "word recognition with dictionary" algorithm producing a ranked dictionary (1). Confidence levels of the ranked dictionary indicate that E. Main St. is the correct stree name. A ZIP+4 Code is created by adding the 4-digil add-oin to the 5-digit ZIP Code $(\mathrm{m})$. The last wo digits of the street number (18) are then added to the $2 \mathrm{IP}+4$ Code to create the final DPC (I).

\section{Performance Evaluation}

The performance of the HWAIS is described in I wo phases, reading the ZIP Code present on the mail piece and assigning a DPC.

The system performance for reading ZIP Codes on a set of 1008 images of live mail addresses is $75.8 \%$ correct with a $1.2 \%$ error rate
(Table I). Since all DPC assignment by our HWAIS relies on ZIP Code determination, the $75.8 \%$ represents an upper bound on DPC atsignnent.

The system performance on DPC was tested on another set of 97.3 images (all addressed to locations inside Buffato). These addresses were chosen because only Buffalo-area DPC directories were available during lesting. ZIP Code recognition performance for these images is $67.4 \%$ correct with $1.11 \%$ errors. The drop in ZIP Code recognition performance was due to the nature of the ZIP Codes (i.e. 7.IP Codes in this set contained many 4 's and 2 's, 14222 was most common; and our digit recognition algorithm performs worst on 4 's and 2 s's.

DPC perfomance varied from $33.1 \%$ correct with $19.6 \%$ enrors 10 $24.4 \%$ correct with $4.3 \%$ errors, depending upon the acceptance threshold level. This pertormance is the current state of the art for fully automatic ststems that determine DPC's from handwritten United States" addresses. White insufticient for practical applications. comtimuing efforts are underway 10 improve performance.

\section{ACKNOWLFDGNFNT}

Wc would like to thank M.-Y. Chen, A. Commike. P. Cullen. J. Favata. R. Fenrich, E. Klemberg. C.-C. Kuan, A. Kundu. D.-S. Lee. C. O Connor. G. Seni. J. Tan and I. Zhou for contribuling ideas and code lowards the HWAIS. This work was supporled by the USPS under Task Order $104230-86 \mathrm{M}-3990$.

\section{REFERENCES}

11) E. Cohen, "Interpreting handw ritten iext in a constrained donlain." Tech. Rep. 92-06. State Univ of New York at Buffalo, Feb. 1992

I2] E. Cohen, J. J. Huil. and S. N. Srihari. "Understinding handwriten text in a structured environment: Determining zip codes from addresses," in Character and Handwriting Recognition: Expandine Froniers, P. S. P. Wang. Ed. Singapore: World Scientific. 1991, pp. 221-264.

[3] N. Otsu. "A threshold selection method from gray-level histograms." IEEE Trans. Syst, Man Cybern., vol. SMC-9, no. 1. pp. 63-66. Jan. 1979 